\title{
Mechanical Tuning of Substrate Integrated Waveguide Filters
}

\author{
Fermín Mira, Member, IEEE, Jordi Mateu, Senior Member, IEEE and Carlos Collado, Senior Member, IEEE
}

\begin{abstract}
This paper presents a novel approach for tuning substrate integrated waveguide (SIW) resonators, realized by placing an additional metalized via-hole on the waveguide cavity. The approach presented here can be applied as a trimming technique, as well as to develop filter designs with tunable center frequencies and tunable bandwidths. Three different filters are designed and implemented, demonstrating excellent trimming, $10 \%$ tuning of the center frequency, and $100 \%$ tuning of the bandwidth respectively.
\end{abstract}

Index Terms-Filters, open-loop slot, resonator, SIW, tuning

\section{INTRODUCTION}

$\mathbf{T}$ HE development of substrate-integrated waveguide (SIW) technology has presented new opportunities for circuits and systems in the microwave and millimeter-wave frequency range. Substrate integrated waveguide structures are based on a synthesized waveguide in a planar dielectric substrate with two rows of metallic vias [1], and exhibit a number of advantages, including easy fabrication, compact size, low loss, complete shielding and easy integration with active devices [2] [3]. Among the wide class of SIW components proposed in the literature, SIW filters have received particular attention, due to the possibility of achieving higher quality-factor [4], compared to classical planar filters in microstrip and coplanar-waveguide technology. This technology also allows for the inclusion of transmission zeros to improve the selectivity at the bandedges [5].

SIW filters are conceptually very similar to filters implemented with waveguide technology, which are conventionally tuned by introducing screws into the resonant cavities and into the coupling apertures. Although the design of SIW and waveguide filters is quite similar, this simple mechanical tuning method is not applicable for SIW technology, due to the compact physical structure. In addition, for SIW technology mechanical tolerances are typically higher, and variations in the dielectric permittivity and thickness of the substrate can introduce additional perturbation in the electromagnetic

Manuscript received July 01, 2014; revised. This work was supported in part by the Spanish Government through grants (TEC- 2012-13897-C03-01, MAT2011-29269-C03-02, by the Generalitat de Catalunya under Grant 2014 SGR 1551, and by the Cluster of Application and Technology Research in Europe on Nanoelectronics (CATRENE) under the project named CORTIF CA116- Coexistence of Radio Frequency Transmission in the Future.

F. Mira is with the Centre Tecnològic de Telecomunicacions de Catalunya, 08860 Castelldefels, Barcelona, Spain (e-mail: fermin.mira@cttc.es).

J. Mateu is with the Department of Signal Theory and Communications, Universitat Politcnica de Catalunya (UPC), Barcelona 08034, Spain and also with the Centre Tecnològic de Telecomunicacions de Catalunya (CTTC), Spain (e-mail: jmateu@tsc.upc.edu)

C. Collado is with the Department of Signal Theory and Communications, Universitat Politcnica de Catalunya (UPC), Barcelona 08034, Spain (e-mail: collado@tsc.upc.edu) response. For this reason, tuning of SIW filters is crucial to compensate for variations in manufacturing and material properties. Moreover, such as tuning capability could be applied as convenient method to change central frequency and bandwidth of the filter.

Electrically tunable resonators have been proposed in [6] where a SIW cavity resonator is combined with a surface mounted varactor to achieve a measured continuous tuning range of $1.2 \%$. In [7], the authors proposed the inclusion of PIN diodes to obtain discrete electrical tuning. Discrete mechanical tuning is proposed in [8] by opening or shortcircuiting a capacitive circular slot, with a tuning range of $5 \%$, or by using MEMS devices [9] with a tuning range of 7\%. A more complex system is presented in [10] which uses a cylinder of plasma in the resonator, but presents only simulated results.

This paper uses the tuning concept for SIW resonators described in [11] to create SIW tunable filters for the first time. In [11] we developed a new concept to tune SIW resonators by including a slot in the top layer and an additional metalized via-hole in the SIW cavity. The dimension of the slot is mechanically controlled by an external metallic flap. Experimental results were presented in a single resonator showing tuning ranges up to $8 \%$. This concept was also later successfully applied for designing tunable oscillators [12]. This work is novel in at least four different respects:

- First, we prove that the same concept used for tunable resonators is applicable to the design of tunable filters.

- Second, we use the same tuning mechanism to control the coupling between resonators as well as the input and output couplings. This allows one to tune the filter bandwidth and also to improve the matching.

- Third, we improve the tuning element performance with the design of a new slot configuration, which provides more accurate control of the resonant frequencies.

- And fourth, new flap configurations are used in different parts of the filter network to control either the filter center frequency, or the couplings between resonators. These new flaps also provide a means for better control over a continuous tuning range, as detailed in Section II.

The application of the tunable mechanism is demonstrated in Section III, which presents the designs and full-wave simulations along with experimental results of three 4-order SIW tunable filters. The first filter uses the tuning elements as trimming elements to adjust the filter response. The second filter provides a tunable bandwidth of $100 \%$ and the third filter offers a central frequency tunability of up to a $10 \%$ range.

Note as well that the tunable center frequency and tunable bandwidth can be combined together in a single filter design. This is illustrated in the design of the second filter, which is 
intended to demonstrate a tunable bandwidth, but which also demonstrates a tunable center frequency of up to $4 \%$.

\section{Tunable SIW Resonator}

This section describes the tunable mechanism concept [11] and describes the tuning elements to be used in the following section for the design of tunable filters.

\section{A. Tunable SIW: Concept}

Figure 1(b) outlines a single resonating SIW cavity fed by microstrip lines. It consists of a conventional SIW resonator with an additional via hole inside of the cavity. Details of the via hole can be seen in Fig. 1 (a). The via hole is partially enclosed by a circular slot connected to the top layer through a metallic contact placed at the angle $\phi$. The via hole is located in the middle of the cavity and displaced from the center by a distance $x$. The existence of this via hole perturbs the electromagnetic field distribution from the one in a uniform SIW resonator and this variation gives rise to a change of the resonant frequency. Details in the field distribution for both side view and bottom view are outlined in Fig. 2. Note that the fundamental resonant frequency of the bare SIW cavity (without via hole) it is essentially defined by its width (w). The inclusion of the via hole results in a reduced effective width of the cavity which results in a higher resonant frequency .

The position of the via hole $x$ and the orientation of the metallic contact $\phi$ modifies the field distribution and affects the effective width of the cavity therefore changing the resonant frequency. In absence of metallic contact, only with the slot, the magnetic wall provides an electric field distribution similar to that of a bare cavity (see Fig. 2(a)), whereas with only the via hole in absence of slot, the electric field is compressed and the resonant frequency is increased (see Fig. 2(c)). From the side view field distribution we can see how in the case of absence of metallic contact the field propagates through the slot as in a capacitance coupling (see Fig. 2(a)), whereas in (see Fig. 2(c)), the boundary conditions of an electric wall forces the field drop to zero and therefore compacting the field as if was a narrower SIW cavity. The inclusion of the metallic contact with angle $\phi$ provides field distributions and resonant frequencies between both the two states previously described. For $\phi=0^{\circ}$ the contact is far from the maximum of the electric field, being this case more similar to the cavity with only the slot (see Fig. 2(b)). On the other hand, for $\phi=180^{\circ}$ the metallic contact is closer to the maximum of the electric field and the field distribution is similar to the cavity without slot (see Fig. 2(d)). As concluded in [11] the tuning frequency range essentially depends on four parameters that define the tuning element. Those parameters are:

- the position of the tuning element in the cavity. Indicated by $x$ in Fig. 1(b).

- The position of the metallic contact between the via hole and the upper (or lower) metallic wall of the cavity. Represented by the angle $\phi$ in Fig. 1(b)).

- The width of the metallic contact $w_{f}$ (see Fig. 1(a)).

- The dimensions of the slot, defined by inner and outer diameters, $d s_{\text {in }}$ and $d s_{\text {out }}$ (see Fig. 1(a)), respectively [11] .

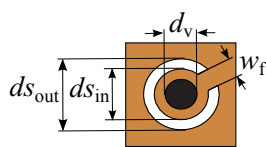

(a) 4

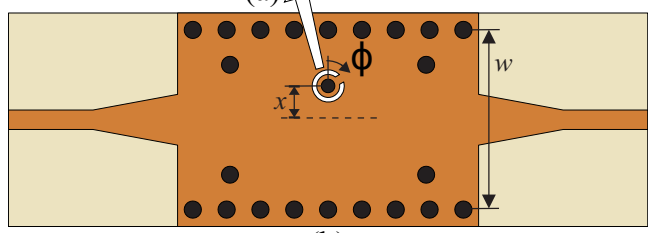

(b)

Fig. 1. (a) Details on the dimensions of the tuning element. (b) SIW cavity with tuning element.

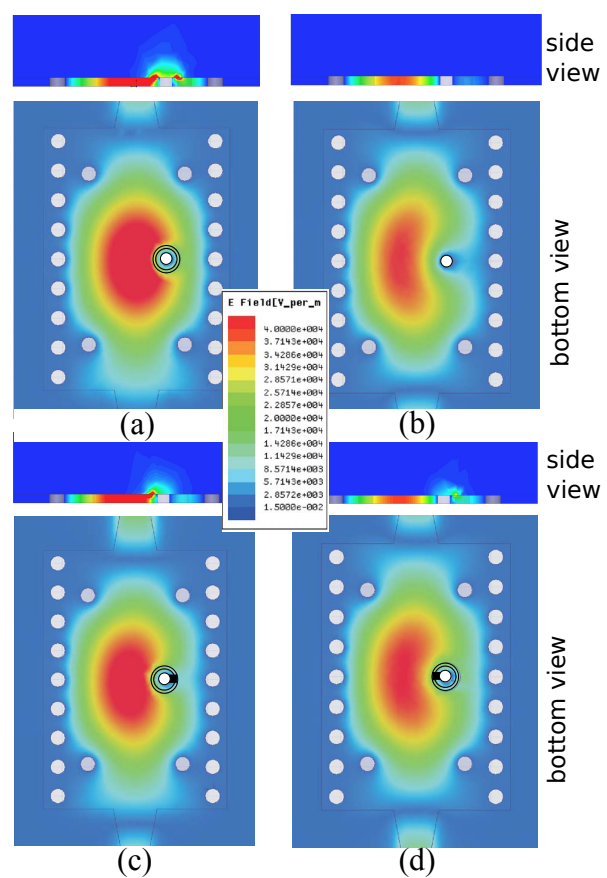

Fig. 2. Electric field distribution on the SIW resonator, for both bottom view and side view. (a) Only slot, (b) contact placed at $\phi=0^{\circ}$, (c) only via hole without slot, (d) contact placed at $\phi=180^{\circ}$.

Additionally, and as pointed out in [11] the dimensions of the via hole of the tuning element ( $d_{v}$ in Fig. 1(a)) also has an effect on the tuning range.

As in [11], the metallic contact will be performed by a metallic flap attached to the tuning screw, which will be mechanically controlled. The orientation of the flap, $\phi$, will therefore be the tuning parameter once have been determined all other dimensions of each tuning element (position of the tuning element, $x$, dimension of the slot, $d s_{i n}$ and $d s_{\text {out }}$, and via hole dimensions, $d_{v}$ ). Also the width of the flap $w_{f}$ must be considered as part of the definition of the tuning element. This concept is presented in Fig. 3, and a similar approach is used in this paper.

\section{B. Tuning elements in SIW filters}

In order to design a tunable filter one also needs to control the coupling between resonators, either because we may desire a variable bandwidth filter or because the coupling between 


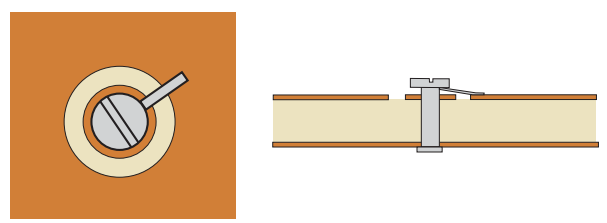

Fig. 3. Outline of the proposed tuning mechanism. Left: top view. Right: side view.

resonators deviates when the resonant frequencies change and we need to recover the initial bandwidth. To do that we introduce a tuning elements as the one in Fig. 1 into the coupling path between resonators, as shown in Fig. 4. Dimensions of the slot and configuration of the metallic flap need to be considered as part of the tunable filter design. In what follows, an assessment of the slot in a two coupled resonator structure is presented. Details of the metallic flaps to be used are also reported in this section.

1) Slot: Figure 4 shows the basic structure used for the design of tunable filters. Two identical resonators (Resonator 1 and Resonator 2) are separated by an inductive coupling window. The two tuning elements placed into the resonators mainly change the resonant frequency, whereas the tuning element placed in the coupling windows mainly modifies the coupling between the two resonant modes. The configuration of Fig. 4 has been simulated with the same material parameters used in [11], and later used in the filter designs. This is a Rogers RO4003 substrate, with relative permittivity of $\epsilon_{r}=3.55$ and $0.813 \mathrm{~mm}$ thickness $(h)$. The loss tangent used for simulation is $\tan \delta=0.0027$.

The dimensions of the cavities in Fig. 4 are set to resonate around $10 \mathrm{GHz}$ when they are isolated. Then the two identical cavities are coupled together through a coupling window. The dimensions of the overall structure result in $w=13 \mathrm{~mm}, l=20$ $\mathrm{mm}$ and $w_{c}=4.9 \mathrm{~mm}$. This results in a coupling coefficient of 0.155 , without the tuning screws, due to a two mode resonating structure with $f_{1}=8.62 \mathrm{GHz}$ and $f_{2}=10.08 \mathrm{GHz}$. The frequencies of the two modes have been obtained through eigenmode analysis. Note that the coupling window affects not only the coupling but also the central frequency of the two mode structure $\left(f_{0}=\sqrt{\left(f_{1} f_{2}\right)}\right)$. This is expected since both cavities are no longer isolated and therefore have different boundary conditions, causing the two cavities to load one another in such a manner as to cause the originally identical mode to split into two different modes [13].

To evaluate the tunable performance of the basic structure of Fig. 4 the central frequency tuning elements $\left(t_{f 1}\right.$ and $\left.t_{f 2}\right)$ are introduced in the middle of the cavity at $l_{1}=5 \mathrm{~mm}$ from the side wall and at $x_{c v}=3.1 \mathrm{~mm}$. The dimensions of the slot are chosen to be $1.7 \mathrm{~mm}$ for the inner diameter $\left(d s_{i n}\right)$ and $2.2 \mathrm{~mm}$ for the outer diameter $\left(d s_{\text {out }}\right)$. The width of the metallic contact ( $w_{f}$ in top inset of Fig. 1) and the diameter of the via hole $d_{v}$ are set to $1 \mathrm{~mm}$. Recall, that the settings above on the central frequency tuning screw position and its dimensions affect the ultimate tuning range.

A coupling tuning element is then introduced in the middle of the coupling window $\left(t_{c 12}\right.$ in Fig. 4). The presence of the tuning element in the coupling window affects the field

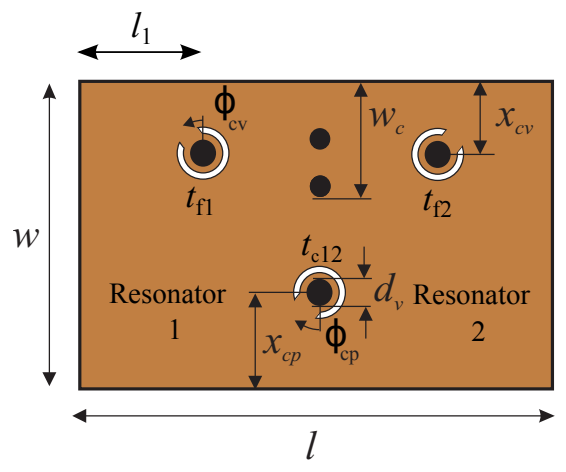

Fig. 4. Basic structure for simulating the tuning mechanisms in filters.

coupled between the two cavities. In turn, the coupling tuning element modifies the boundary conditions of the resonators and thus also affects the central frequency. In spite of that, we can fairly consider that the $\phi_{c v}$ angle would tailor the central frequency of the resonators and $\phi_{c p}$ would control the coupling between them.

The effects of $\phi_{c v}$ and $\phi_{c p}$ are summarized in Table I for two values of the angles $0^{\circ}$ and $180^{\circ}$, two different diameters of the via hole $d_{v}$ and two different positions $x_{c p}$. Note that with these angles we consider the whole range of tuning, and all the values in between can be achieved continuously. The table indicates the values of the two fundamental frequencies $f_{1}$ and $f_{2}$ for each combination of $\phi_{c v}$ and $\phi_{c p}$ and the resulting coupling coefficients, $m$. The value of $m$ has been calculated following the well known expression, $m=\left(f_{2}^{2}-f_{1}^{2}\right) /\left(f_{1}^{2}+f_{2}^{2}\right)$ [13] and ranges from 0.044 to 0.163 . From the table we can generally confirm that for any of the tuning element dimensions and positions, when the angle of the coupling flap $\left(\phi_{c p}\right)$ is fixed the value of the coupling coefficient $m$ barely changes, and only the central frequency is affected.

According to the results of Table I, the range of tuning is higher as $d_{v}$ increases and when the coupling tuning element moves out from the center of the coupling window $\left(x_{c p}=2\right)$. For $d_{v}=1 \mathrm{~mm}$ the coupling range is larger than $30 \%$, and for $d_{v}=2 \mathrm{~mm}$ it can be increased up to $90 \%$. Thus, depending on the application, we chose the diameter of the via hole and its position in order to get the required tuning range. This demonstrates the usefulness of this tuning approach also for controlling the coupling between resonators. Note that modification of this configuration, as two tuning elements in the coupling window or two tuning elements in the cavity would be also used for further controlling the tuning performance.

In order to further illustrate the design and placement of the coupling tuning element $\left(t_{c 12}\right)$ on the coupling window $\left(x_{c p}\right)$, Fig. 5 depicts the value of the coupling coefficient $m$ for several positions of the tuning element, ranging from $x_{c p}=1.5$ $\mathrm{mm}$ to $x_{c p}=6.5 \mathrm{~mm}$ as a function of the angle $\phi_{c p}$ of the metallic contact. For this parameterization the width of the metallic contact $\left(w_{f}\right)$ and the diameter of the via hole $\left(d_{v}\right)$ are fixed to $1 \mathrm{~mm}$. The results in Fig. 5 show how, for positions where the tuning element is closer to the lateral wall ( $x_{c p}$ small), the coupling decreases when the angle of 


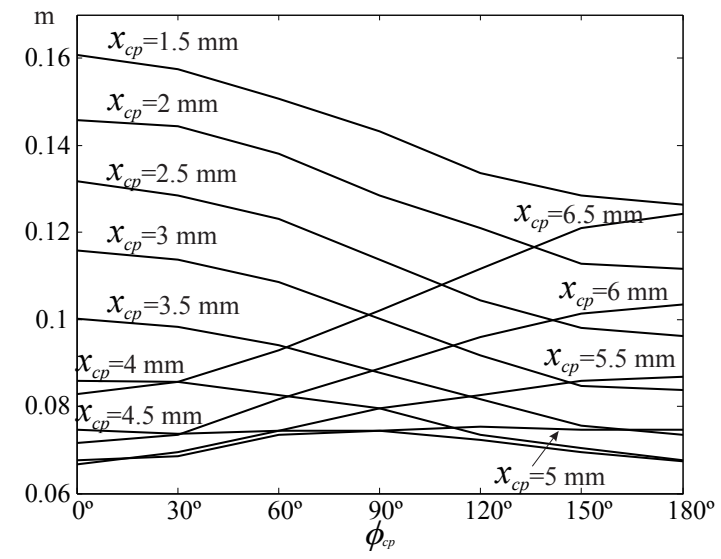

Fig. 5. Coupling between the two cavities of Fig. 4 for several positions of the tuning element and as a function of the angle position of the metallic contact.

the metallic flap increases. This has the effect of making the coupling window narrower. On the other hand when the position of the coupling tuning element gets closer to the coupling wall, the coupling window is narrower for smaller angles. Note that this type of parameterization it is very useful in a design process since it sets the position of the tuning element for a desired range of tunability. From this assessment it might be also concluded that for a given via hole $\left(d_{v}\right)$, slot $\left(d s_{i n}\right.$ and $\left.d s_{\text {out }}\right)$ and metallic flap dimensions $\left(w_{f}\right)$ of the coupling tuning element the tuning range (difference between the maximum and minimum coupling values) barely changes and the achievable coupling is controlled by the position of the coupling tuning element $\left(x_{c p}\right)$.

2) Flap: In contrast to our previous work, where a simple screw with a single straight metallic flap was used (see details in Fig. 3), in this work several flaps have been considered in order to offer a better control of the tuning requirements for a filter implementation. Figure 6 shows three different configurations of the flap. The flaps would be attached to the tuning screw and to the top layer of the SIW cavity defining therefore the length of the slot (or width of the metallic contact $\left.w_{f}\right)$.

These flaps will be used in the filters presented in the following section. Although further details will be given, the main differences between the flaps of Fig. 6 are the width of the flap and position. The first flap on the left is designed for half slots and short tuning range, where the slot progressively covers all the slot. The third flap, in contrast, has a small metallic part, covering an small part of the slot, and then resulting in a wide tuning range. Finally, the flap in the middle is designed to produce a symmetrical coverage of the slot, which shows to be convenient when a symmetrical input and output reflection coefficients are required.

\section{TUnABLE SIW Filter}

This section uses the tuning elements above for further development of the tuning concept. To do that, several tuning effects have been applied into three filter designs. Initially, all filters (without tuning elements) consist in conventional four-pole Chebyshev filters centered at $10 \mathrm{GHz}$ with $8 \%$ of
TABLE I

COUPLING AND RESONANT FREQUENCIES FOR THE BASIC STRUCTURES OF FIG. 4

\begin{tabular}{|c|c|c|c|c|c|c|}
\hline $\begin{array}{c}x_{c p} \\
(\mathrm{~mm})\end{array}$ & $\begin{array}{c}d_{v} \\
(\mathrm{~mm})\end{array}$ & $\phi_{c v}$ & $\phi_{c p}$ & $f_{1}(\mathrm{GHz})$ & $f_{2}(\mathrm{GHz})$ & $\mathrm{m}$ \\
\hline 4 & 1 & $0^{\circ}$ & $180^{\circ}$ & 9.86 & 10.55 & 0.068 \\
\hline 4 & 1 & $180^{\circ}$ & $180^{\circ}$ & 10.26 & 11.01 & 0.070 \\
\hline 4 & 1 & $0^{\circ}$ & $0^{\circ}$ & 9.68 & 10.55 & 0.086 \\
\hline 4 & 1 & $180^{\circ}$ & $0^{\circ}$ & 10.06 & 11.00 & 0.089 \\
\hline 2 & 1 & $0^{\circ}$ & $180^{\circ}$ & 9.45 & 10.56 & 0.111 \\
\hline 2 & 1 & $180^{\circ}$ & $180^{\circ}$ & 9.81 & 11.02 & 0.116 \\
\hline 2 & 1 & $0^{\circ}$ & $0^{\circ}$ & 9.10 & 10.55 & 0.147 \\
\hline 2 & 1 & $180^{\circ}$ & $0^{\circ}$ & 9.41 & 11.00 & 0.155 \\
\hline 4 & 2 & $0^{\circ}$ & $180^{\circ}$ & 10.17 & 10.63 & 0.044 \\
\hline 4 & 2 & $180^{\circ}$ & $180^{\circ}$ & 10.57 & 11.08 & 0.047 \\
\hline 4 & 2 & $0^{\circ}$ & $0^{\circ}$ & 9.78 & 10.60 & 0.080 \\
\hline 4 & 2 & $180^{\circ}$ & $0^{\circ}$ & 10.16 & 11.03 & 0.082 \\
\hline 2 & 2 & $0^{\circ}$ & $180^{\circ}$ & 9.77 & 10.61 & 0.082 \\
\hline 2 & 2 & $180^{\circ}$ & $180^{\circ}$ & 10.16 & 11.06 & 0.085 \\
\hline 2 & 2 & $0^{\circ}$ & $0^{\circ}$ & 9.02 & 10.55 & 0.155 \\
\hline 2 & 2 & $180^{\circ}$ & $0^{\circ}$ & 9.32 & 10.99 & 0.163 \\
\hline
\end{tabular}

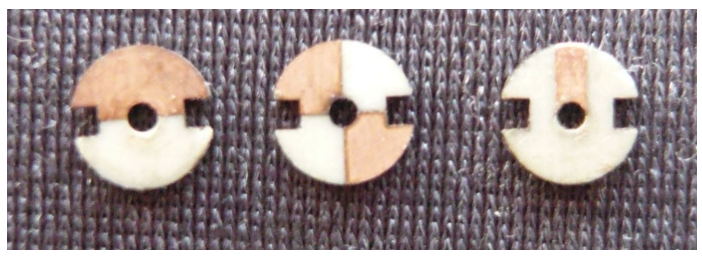

Fig. 6. Flaps used for tuning the filters.

fractional bandwidth (FBW), with targered return losses of $20 \mathrm{~dB}$. This sets the required coupling between resonators and the coupling between the input/output ports to the first/last resonators by means of the coupling matrix. For a 4 order Chebyshev filter with $20 \mathrm{~dB}$ of return losses the resulting normalized coupling matrix is :

$$
\left(\begin{array}{llllll}
0 & 1.152 & 0 & 0 & 0 & 0 \\
1.152 & 0 & 1.041 & 0 & 0 & 0 \\
0 & 1.041 & 0 & 0.772 & 0 & 0 \\
0 & 0 & 0.772 & 0 & 1.041 & 0 \\
0 & 0 & 0 & 1.041 & 0 & 1.152 \\
0 & 0 & 0 & 0 & 1.152 & 0
\end{array}\right)
$$

which is then used to obtain the coupling coefficients $m$ by multiplying for the FBW. This results in the required coupling coefficients ranging from 0.09 to 0.06 . Note that those values are similars to the ones outlined in Fig. 5. Also mention that they do not have to be equal since the coupling window is not the same than in the filter designs. The dimensions of the coupling windows on the resonator topologies have been designed to achieve the couplings obtained from the synthesis.

Tuning elements for the central frequency of each resonators, for couplings between each pair of consecutive res- 
onators, and also for the input and output coupling have then been included into the filter configurations. To illustrate this we can see the picture of the filter in Fig. 7. This picture numbers each cavity (1, 2, 3 and 4), with their corresponding frequency tuning elements, $t_{f i}$, the coupling element between cavities $i$ and $j, t_{c i j}$, and also between the source and the first cavity, $t_{c S 1}$, and the coupling element between the last cavity and the load, $t_{c 4 L}$. Dimensions and positions of these elements have been selected for the desired functionality. This is for trimming purposes, bandwidth tunability and central frequency tunability. Details on the three designs appear below.

\section{A. Trimmed filter}

In the first example the tuning approach is used as a trimming technique in order to recover the desired filter performance, initially deviated due to fabrication tolerances.

Since the expected deviation is small, the placement and design of the tuning screws are set for a short turning range, enough for small adjustments. Note that as stated in [11] small tuning range results in fairly constant $Q$ values, which ensures the flatness and selectivity of the filter.

Figure 7 shows the fabricated filter without the tuning screws. The half slots combined with the flaps of Fig. 6 (first on the left) allows for a small tuning while preserving most of the $Q$ factor. Those tuning elements are placed close to the metallic wall, also for the purpose of barely affecting the initial field distribution, and therefore with a small effect on the resonant frequency.

The tuning elements in this case are all equal, but the one in the middle, $t_{c 23}$. The first and last tuning elements ( $t_{c s 1}$ and $t_{c 4 L}$ ) control the input and output couplings of the filter and are highly related with the reflective coefficients. The tuning element controlling the coupling between resonator 2 and 3 $\left(t_{c 23}\right)$ is set in the middle of the filter and has been chosen to be different just for preserving the symmetry on the structure. For the same reason this slot is using the second flap in Fig. 6 (the flap in the middle). Diameter of the via hole of the tuning elements $\left(d_{v}\right)$, and the inner and outer diameter of the slot $\left(d s_{\text {in }}\right.$ and $\left.d s_{\text {out }}\right)$ are $1 \mathrm{~mm}, 1.7 \mathrm{~mm}$ and $2.2 \mathrm{~mm}$, respectively.

Figure 8 shows the response of the filter before and after the trimming process. Dashed line shows the full-wave simulated response (Ansys HFSS v15) of the initial filter (without tuning elements) and therefore the expected from the measured response. In grey we show the measured response of the filter when the tuning elements are set to cover half the slots on Fig. 7. Note that this should result in the design response (in dash), however some disagreement can be observed in the reflective response. The trimming process then starts by moving the tuning screws (see details in the inset of Fig. 8) and uncover part of the slot. The resulting response after the trimming process is depicted in black solid line in Fig. 8. We can see a fairly good agreement between the designed and the filter response after trimming. Note as well that the input and output reflection coefficients reveal symmetry as expected from the initial design and ensured from the slot and flap distribution and configuration along to the filter topology.

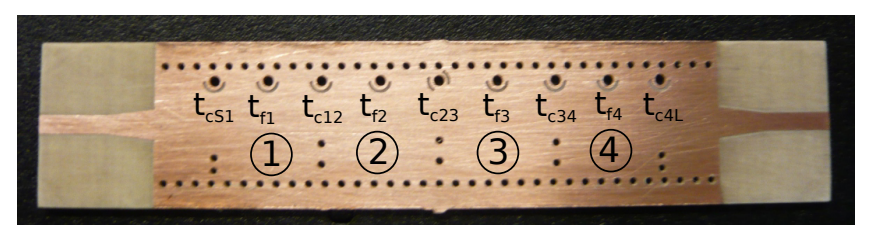

Fig. 7. Tunable filter without the tuning screws.

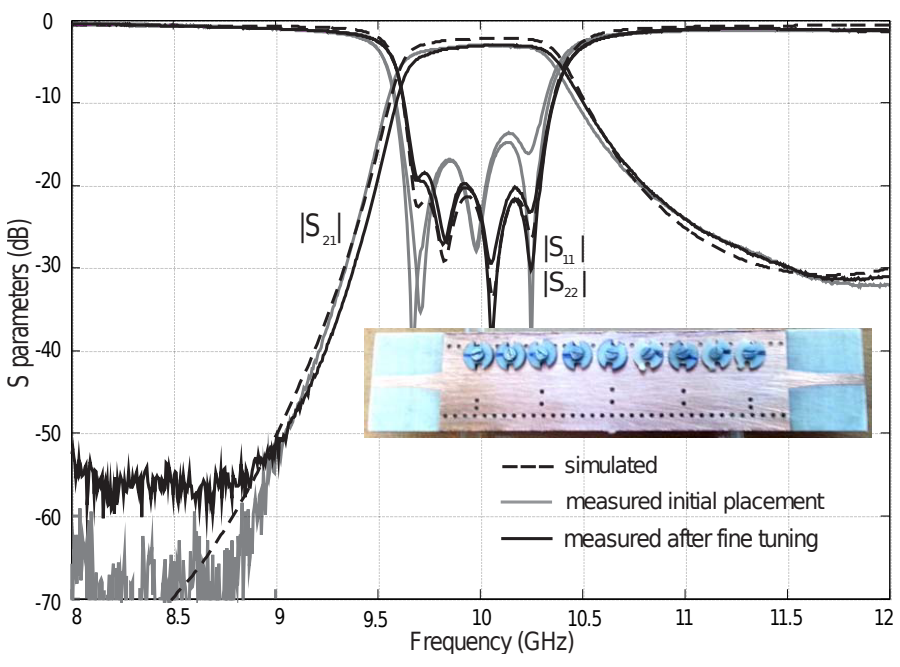

Fig. 8. Simulated and measured results of the tunable filter.

\section{B. Bandwidth tunable filter}

The second filter configuration is outlined in Fig. 9, with tuning screws of $1 \mathrm{~mm}$ of diameter to obtain a medium tuning range, both on bandwidth and/or on the central frequency. In contrast with previous case where only half slots were employed, in this case full slots are used in order to increase the tuning range. As stated above it also consists on a fourpole order filter centered at $10 \mathrm{GHz}$ approximately. In contrast from previous filter of Fig. 7, the coupling windows between resonators have been differently arranged only for a better location of the tuning elements. The slots corresponding to these central frequency tuning elements $\left(t_{f i}\right)$ are placed in the middle along the cavity and close to the metallic wall and in the side where the coupling wall exists. This is convenient to reduce the effect of these tuning elements to the coupling between resonators. On the other hand the coupling tuning elements $\left(t_{c i j}\right)$ are located along the coupling wall, as outlined in Fig. 4. For the input and output coupling $\left(t_{c S 1}\right.$ and $\left.t_{c 4 L}\right)$ and for the coupling between the first and the second resonators $\left(t_{c 12}\right)$ and for the coupling between the third and fourth resonators $\left(t_{c 34}\right)$ a single tuning element is used to control the coupling. Whereas for the coupling between the second and third resonators $\left(t_{c 23}\right)$ two tuning elements are symmetrically located for the control of such coupling. Note also that they are half slots. The main reason for that, as in previous case, is preserving the symmetry of the whole structure, even when tuning elements are inserted.

The tuning elements are then finalized by the inclusion of the metallic flaps. In this case and in order to obtain a wider tuning range, the flap configuration shown in the right side of Fig. 6 is used. The width of the flap is chosen to be $w_{f}=1$ 
$\mathrm{mm}$, equal to the diameter of the tuning screws in order to optimize the tuning range. The inner and outer diameters of the slot $\left(d s_{\text {in }}\right.$ and $\left.d s_{\text {out }}\right)$ are chosen as in previous filter.

The tuning screws are then used to prove a variable bandwidth. Figure 10 shows the simulated and measured responses for the case where the maximum bandwidth can be achieved (grey traces in Fig. 10) and with the minimum bandwidth (black traces in Fig. 10). At this point it is worth to mention that due to the symmetry of filter configuration the position and angle $\left(\phi_{i}\right)$ of the tuning elements are set to also be symmetrical. This is $t_{c S 1}=t_{c 4 L}, t_{c 12}=t_{c 34}, t_{f 1}=t_{f 4}$ and $t_{f 2}=t_{f 3}$. Table II shows the angle of the tuning elements for both configurations, where $0^{\circ}$ is for the flap pointing outside the filter and turns towards the nearest port, thus preserving the symmetry. The terms $\phi_{c v 1}$ and $\phi_{c v 2}$ indicate the rotation angle of the central frequency tuning screws of resonators 1 and 2 , respectively. On the other hand the term $\phi_{c p s 1}$ corresponds to the rotation angle of tuning element setting the input external coupling. Finally the terms $\phi_{c p 12}$ and $\phi_{c p 23}$ give the angle of the tuning elements for the coupling between the first and second resonator and between the second and third resonator, respectively. Note that the structure includes two tuning elements in the center for coupling between the second and third resonator, pointing in opposite directions in order to preserve the symmetry. The position of the metallic flaps have been marked on the top part of the tuning screw in order to know their positions while tuning. From this view we can confirm the symmetry between the position of the tuning screws.

The values detailed in Table II, for the tunable bandwidth filter (second and third rows), indicate that for the narrower bandwidth filter the coupling window should be narrower, whereas for the broader bandwidth filter the window should be wider. Values of the table also reveal that the tuning elements corresponding to the central frequency of the resonators are barely moved and only for sake of preserving the central frequency and good reflection coefficient.

According to simulations and measured results the filter bandwidth can be tuned up to a $100 \%$, so the bandwidth of the filter could be doubled. Simulated and measured response agree fairly well in both case.

As illustrative example, we use this filter to evaluate the possibility of applying both functionalities central frequency and bandwidth tunability in a single design. Figure 11 shows simulated results of how the central frequency can be shifted starting from the filter with higher bandwidth. With this layout a tuning range of the central frequency of $4 \%$ could be obtained. For higher tuning ranges we would need to introduce larger tuning screws in the resonators.

\section{Frequency tuned filter}

The last filter has been designed to offer a wider tuning range of the central frequency. As indicated in the example above this requires of larger tuning elements for the central frequency of each resonator. This new filter configuration is outlined in Fig. 12. In this case we preserve the same topology and dimension of the previous filter (Fig. 9) but the diameter

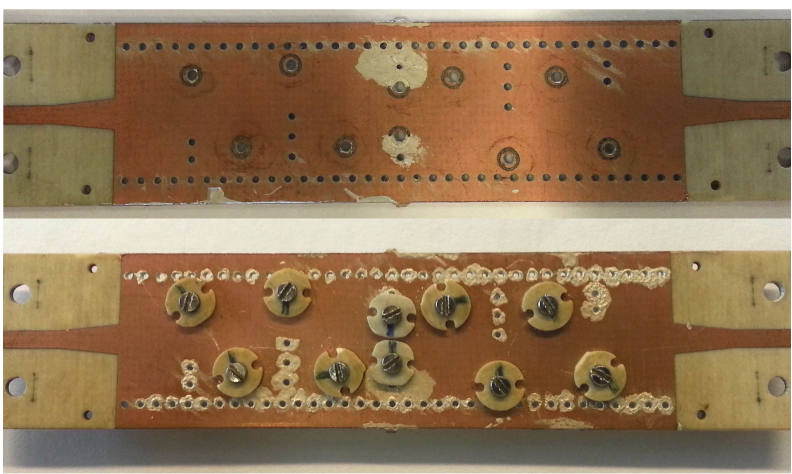

Fig. 9. Filter with and without the tuning screws.

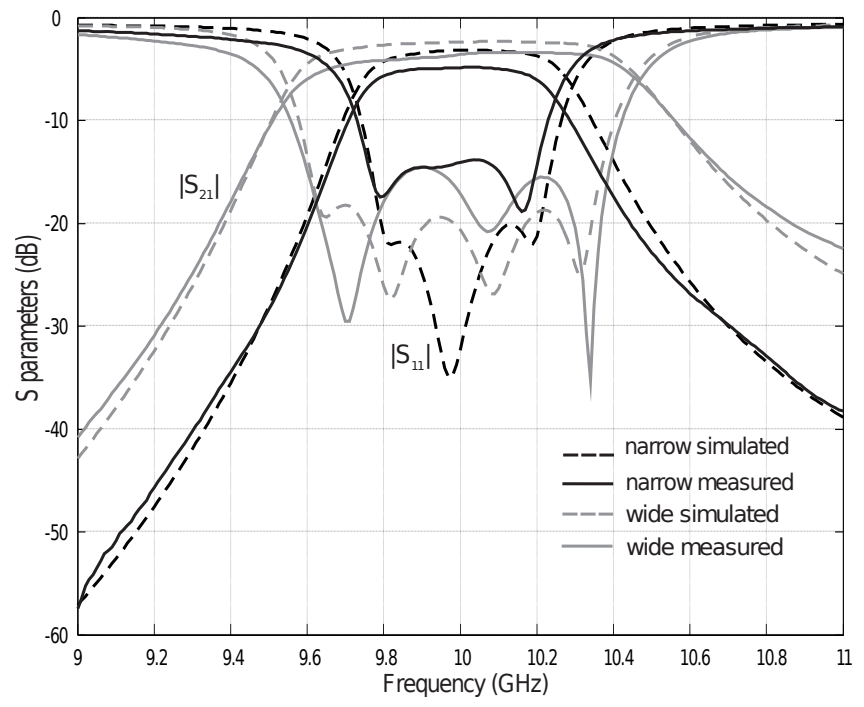

Fig. 10. Fabricated filter with tuning screws for bandwidth variation (simulated and measured).

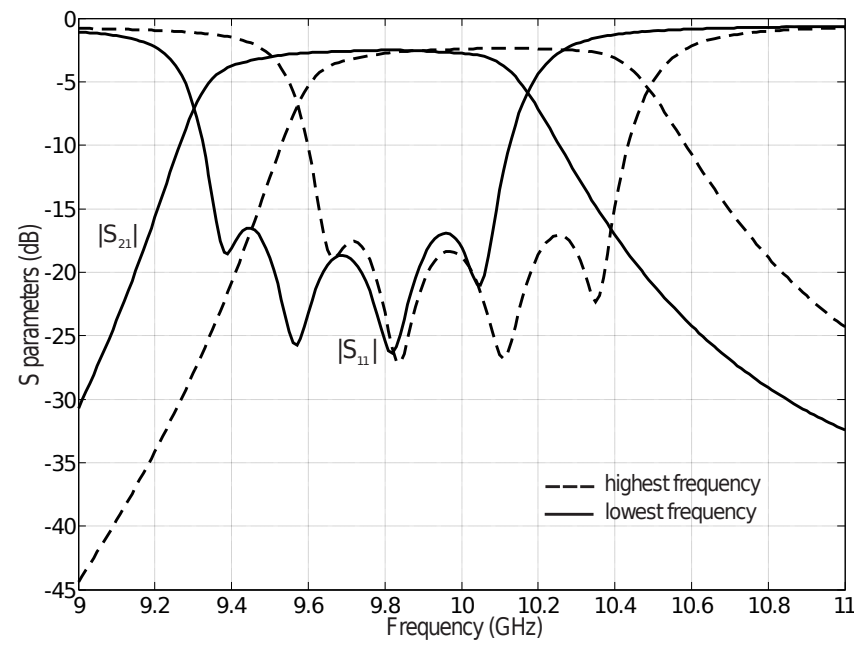

Fig. 11. Variation of the central frequency for the filter of Fig. 9.

of central frequency tuning screws. The diameter of the via hole is $d_{v}=2.5 \mathrm{~mm}$, with an inner and outer slot radius of $d s_{\text {in }}=3.2 \mathrm{~mm}$ and $d s_{\text {out }}=3.7 \mathrm{~mm}$, respectively. The objective of this configuration is to shift the central frequency up to $10 \%$. 


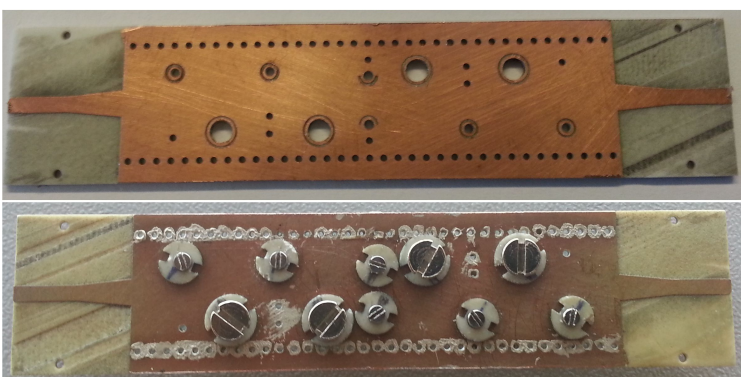

Fig. 12. Filter with and without the tuning screws for central frequency variation.

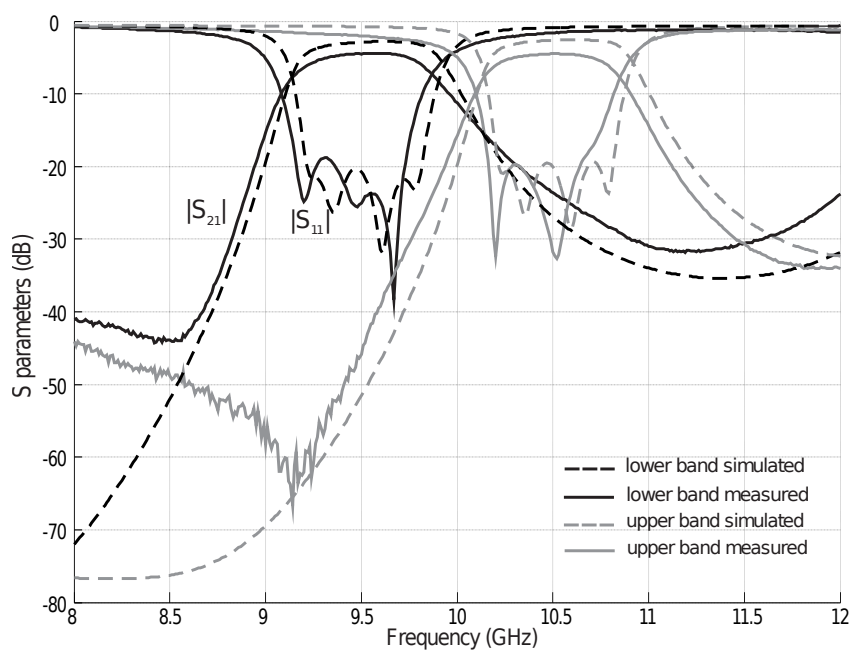

Fig. 13. Fabricated filter with tuning screws for central frequency variation (simulated and measured).

TABLE II

ANGLES FOR THE FILTERS

\begin{tabular}{|l|l|l|l|l|l|}
\hline & $\phi_{c v 1}$ & $\phi_{c v 2}$ & $\phi_{c p s 1}$ & $\phi_{c p 12}$ & $\phi_{c p 23}$ \\
\hline Filter BW narrow & $118^{\circ}$ & $60^{\circ}$ & $35^{\circ}$ & $180^{\circ}$ & $180^{\circ}$ \\
\hline Filter BW wide & $-153^{\circ}$ & $-129^{\circ}$ & $0^{\circ}$ & $10^{\circ}$ & $0^{\circ}$ \\
\hline Filter band 9.5 & $75^{\circ}$ & $66^{\circ}$ & $110^{\circ}$ & $82^{\circ}$ & $185^{\circ}$ \\
\hline Filter band 10.5 & $164^{\circ}$ & $180^{\circ}$ & $175^{\circ}$ & $160^{\circ}$ & $130^{\circ}$ \\
\hline
\end{tabular}

Figure 13 shows the simulated and measured filter responses of two stages of the tunable filter. The passband of the filter has been shifted up to a $10 \%$ in this case, from $9.5 \mathrm{GHz}$ to $10.5 \mathrm{GHz}$. Measured and simulated responses show the same range of tunability and both responses agree fairly well in both tuning stages. The values of the rotating angles for both stages are detailed in rows four and five of Table II. These values reveal that the frequency tuning has been mainly obtained by shifting the position of the central frequency tuning screws, additional adjustment of the coupling tuning screws is required in order to preserve the bandwidth and good matching throughout the whole bandpass of the filter.

Note that, although only two stages have been shown in the previous two examples a continuous tunability is possible.

\section{CONCLUSION}

The paper demonstrates the use of open slots and tuning screws as a useful approach for the tunability of SIW structures. Accurate analysis of the role of each tuning element in coupled resonator structures has been reported. Then several examples of filters have been presented to demonstrate the usefulness of the approach in a trimming problem, in a tunable bandwidth filter and in a tunable central frequency filter. The designs presented in the paper show up to a $100 \%$ of bandwidth increment and a 10\% of tunability of the central frequency. A single filtering structure has been also used to demonstrate the three functionalities at the same time achieving a filter with a bandwidth tunability up to a $100 \%$ and a central frequency tuning of $4 \%$. Additionally and taking into account that the tolerances in the dielectric constant and fabrication process may produce lack of accuracy in the measured response, the inclusion of these tuning screws have also been proved to be useful as a trimming element to obtain an improved performance of the filters. In this way, we avoid the repetition in the fabrication of filters to comply with the desired response or the necessity of very accurate and expensive fabrication technologies.

Note moreover that the same concept could be directly applied to more compact and integrated configuration such as MEMS as a mechanical tuning component.

\section{ACKNOWLEDGMENT}

The authors would like to thank Dr. James C. Booth from National Institute of Standards and Technology for his help on the revision of the paper.

\section{REFERENCES}

[1] U. Hiroshi, T. Takeshi, and M. Fujii, "Development of a laminated waveguide", IEEE Trans. Microw. Theory Techn., vol. 46, no. 12, pp. 2438-2443, Feb. 1998.

[2] D. Deslandes and K. Wu, "Accurate modeling, wave mechanisms, and design considerations of a substrate integrated waveguide", IEEE Trans. Microw. Theory Techn., vol. 54, no. 6, pp. 2516-2526, June 2006.

[3] M. Bozzi, L. Perregrini, and K. Wu, "Modeling of conductor, dielectric and radiation losses in substrate integrated waveguide by the boundary integral-resonant mode expansion method", IEEE Trans. Microw. Theory Techn., vol. 56, no. 12, pp. 3153-3161, Dec. 2008.

[4] M. Bozzi, M. Pasian, L. Perregrini and K. Wu, "On the losses in substrate integrated waveguides and cavities", International Journal of Microwave and Wireless Technologies, vol. 1, no. 5, pp. 395-401, Oct. 2009.

[5] X.P. Chen and K. Wu, "Substrate Integrated Waveguide Cross-Coupled Filter With Negative Coupling Structure", IEEE Trans. Microw. Theory Techn., vol. 56, no. 1, pp. 142-149, Dec. 2008.

[6] F. He, X. Chen, K. Wu and W. Hong, "Electrically tunable substrate integrated waveguide reflective cavity resonator", in Asia-Pacific Confrence APMC, Dec. 2009, pp. 119-122.

[7] M. Armendariz, V. Sekar, K. Entesari, "Tunable SIW bandpass filters with PIN diodes", in Proc. $40^{\text {th }}$ European Microw. Conf., Paris, Sept. 2010, pp. 830-833.

[8] J.C. Bohorquez, B. Potelon, C. Person, E. Rius, C. Quendo, G. Tanne and E. Fourn, "Reconfigurable planar SIW cavity resonator and filter", in IEEE MTT-S International Microwave Symposium Digest, San Francisco, CA, June 2006, pp. 947-950.

[9] W. Gautier, A. Stehle, B. Schoenlinner, V. Ziegler, U. Prechtel and W. Menzel, "RF-MEMS tunable filters on low-loss LTCC substrate for UAV data-link", in Proc. $39^{\text {th }}$ European Microw. Conf., Roma, Sept. 2009, pp. $1700-1703$.

[10] A. Djermoun, G. Prigent, N. Raveu, T. Callegari, "Widely tunable high-Q SIW filter using plasma material", in IEEE MTT-S International Microwave Symposium Digest, Anaheim, CA, May 2010, pp. 1484-1486.

[11] F. Mira, J. Mateu and C. Collado "Mechanical Tuning of Substrate Integrated Waveguide Resonators", IEEE Microwave and Wireless components letters, vol. 22, no. 9, pp. 447-449, Sept. 2012.

[12] A. Collado, F. Mira and A. Georgiadis "Mechanically Tunable Substrate Integrated Waveguide (SIW) Cavity based Oscillator", IEEE Microwave and Wireless components letters, vol. 23, no. 9, pp. 489-491, Sept. 2013.

[13] J. S. Hong, M. J. Lancaster "Microstrip filters for RF/microwave applications", John Wiley \& Sons, 2001. 


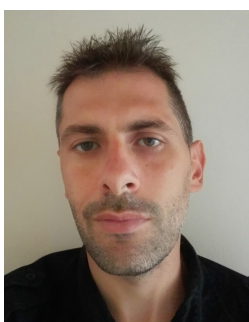

Fermín Mira received the Telecommunications Engineering degree and the $\mathrm{PhD}$ degree in Telecommunications from the Universidad Politecnica de Valencia in 2000 and 2005, respectively. In 2001, he joined the Department of Electronics, Universita degli Studi di Pavia, Italy, where he was a PreDoctoral Fellow (2001-2004) involved with a research project financed by the European Community under the framework of a Maricurie Action of the 5th Program Marco "Millimeter-Wave and Microwave Components Design Framework for Ground and Space Multimedia Network (MMCODEF)", aimed to the development of a CAD tools for the design passive microwave components and with the participation of the European Space Agency. In May 2004, he joined the Department of Communications, Universidad Politecnica de Valencia, working toward his PhD degree. In January 2006 he joined the Centre Tecnologic de Telecomunicacions de Catalunya (CTTC) as researcher in the division of Communication Technologies. His current research interests include numerical methods for electromagnetic modelling of microwave components, and design and fabrication of microwave devices and systems, especially in substrate integrated waveguide (SIW) technology.

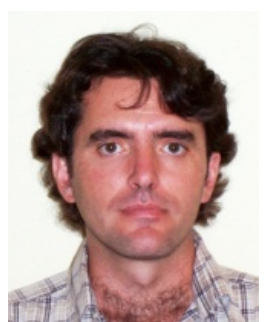

Carlos Collado received the Telecommunication Engineering and $\mathrm{Ph} . \mathrm{D}$. degrees from Universitat Politecnica de Catalunya (UPC), Barcelona, in 1995 and 2001, respectively. He also holds an M.S. on Biomedical Engineering (2002). He joined the faculty at UPC in 1998, and he became Associate Professor in 2005. Since Nov-2005 to Jan-2008 he was vice-dean of the Technical School of Castelldefels (EPSC) of UPC and responsible of the Telecommunication and Aeronautic Engineering degrees. He was a visiting researcher at the University of California Irvine in 2004 and a guest researcher at the National Institute of Standards and Technology (NIST), Boulder in 2009-2010. His primary research interests include microwave devices and systems, superconducting devices, study of efficient methods for the analysis of nonlinear effects in communication systems and characterization of the nonlinear behavior of electro-acoustic devices. He is author or co-author of more than 50 scientific and technical journal papers, and more than 60 contributions to international conferences.

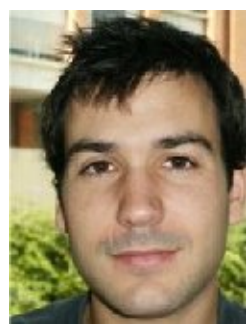

Jordi Mateu (M'03 - SM'10) received the TelecommunicationEngineering and $\mathrm{Ph} . \mathrm{D}$. degrees from the Universitat Politecnica de Catalunya (UPC), Barcelona, in 1999 and 2003, respectively. He is an Associate professor in the Signal Theory and Communications Department, UPC, and Senior Researcher Associate at CTTC. From May to August 2001, he was a Visiting Researcher with Superconductor Technologies Inc., Santa Barbara, CA. From October 2002 to August 2005, he was research staff and coordinator of Communication Subsystems with CTTC, Spain. Since September 2004, he has held several guest researcher appointments at the National Institute of Standards and Technology (NIST), Boulder, CO, where he was a Fulbright Research Fellow from 2005 to 2006. In July 2006, he was a visiting Researcher at Lincoln Laboratory, MIT. From September 2003 to August 2005, he was a part-time Assistant Professor at Universitat Autonoma de Barcelona. In summer 1999, after graduation, he held a trainee engineer position in the Investment Technology department, Gillette, UK. He has authored or co-authored more than 45 papers in international journals, more than 60 contributions in international conferences, and three book chapters, and holds two patents. He collaborated and lead several research projects for national and international public and private organizations and companies. His primary interest includes microwave devices and systems and characterization and modeling of new electronic materials, including ferroelectrics, magnetoelectric, superconductors, and acoustic devices. His recent research also includes the synthesis, design, and development of novel microwave filtering structures.

Dr. Mateu was the recipient of the 2004 prize for the best doctoral thesis in Fundamental and Basic Technologies for Information and Communications, awarded by COIT (Colegio Oficial de Ingenieros de Telecomunicacion) and AEIT (Asociacion Espanola de Ingenieros de Telecomunicacion). He was also the recipient of a Postdoctoral Fulbright Research Fellowship and an Occasional Lecturer Award for visiting MIT. He was second ranked in a Ramon y Cajal Contract (2005), in the area of Electrical and Communication Technologies, a National Program for promoting outstanding Young researchers. From February 2011 to June 2012, he was Vice-Dean at the Telecommunication and Aerospace Engineering School of UPC. He is a reviewer of several journals and international conferences. 